\section{Ciencia Económica}

Revista electrónica de la Facultad de Economía de la Universidad Nacional Autónoma de México

UNAM

Enrique Graue Wiechers

Rector

Leonardo Lomelí Vanegas

Secretario General

Leopoldo Silva Gutiérrez

Secretario Administrativo

Alberto Ken Oyama Nakagawa

Secretario de Desarrollo Institucional

César Iván Astudillo Reyes

Secretario de Atención

a la Comunidad Universitaria

Mónica González Contró

Abogada General

FACULTAD DE ECONOMÍA

Eduardo Vega López Director

Rosa Carmina Ramírez Contreras Secretaria General

Porfirio Díaz Rodríguez

Secretario Administrativo

Ricardo Iglesias Flores

Coordinador de Publicaciones

CIENCIA ECONÓMICA

Mauro Rodríguez García Director

Karina Navarrete Pérez

Secretaria Técnica

Comité Editorial

Andrés Blancas Neria (Instituto de Investigaciones Económicas, UNAM)

Jorge Ibarra Consejo

(Facultad de Economía, UNAM)

Carlos Ibarra Niño

(Universidad de las Américas, Puebla)

Carlos Maya Ambía

(Universidad de Guadalajara)

Gustavo Vargas Sánchez

(Facultad de Economía, UNAM)

Fundadores

Jorge Carreto Sanguinés

Irma Escárcega Aguirre

Rogelio Huerta Quintanilla

Mauro Rodríguez García

Paulo Scheinvar Akcelrad†
Ibarra Consejo, J., 2015.

Materiales de apoyo a la docencia
Fecha de publicación: 2 de febrero de 2016

Facultad de Economía

Año 4 • no. 6 enero-junio de 2015 


\section{La determinación del producto y el empleo totales: un modelo poskeynesiano elemental}

Jorge Ibarra Consejo

Facultad de Economía, Universidad Nacional Autónoma de México (UNAM) <ibacons@unam.mx>

doi: 10.22201/fe.24484962e.2015.v4n6.a3

D os elementos fundamentales del enfoque poskeynesiano que permiten explicar el tamaño del producto total y que son compartidos por otros enfoques heterodoxos son: 1) la importancia que se le da a la demanda agregada en la determinación del producto y 2) la decantación de la distribución del ingreso en el terreno del juego de poder que se da entre perceptores de ingresos laborales y perceptores del excedente por encima del costo laboral. Este juego de poder se desarrolla en dos momentos: el de la negociación salarial entre empleados y empleadores y el de fijación de precios por parte de los productores.

A continuación se presenta un modelo elemental de determinación del nivel de precios, la distribución del ingreso, el producto total y el empleo. Se consideran tanto las condiciones de demanda como las condiciones de oferta, reuniéndolas a través de la relación existente entre la fijación de los precios y la distribución del ingreso. La determinación del producto y el empleo totales estará en función del tamaño de la demanda agregada.

Algunos aspectos del enfoque poskeynesiano se resaltan mejor en un modelo básico construido en términos de un escenario sumamente simplificado como el que aquí se utiliza. Para ello haremos los siguientes supuestos:

1. Se trata de una economía cerrada y sin gobierno. Es decir, existen únicamente empresas privadas, quienes reciben el excedente por encima del costo laboral, y sus trabajadores, quienes reciben los salarios. EI excedente es el monto que deberá ser compartido por las ganancias empresariales, los intereses y los arrendamientos, es decir, los ingresos derivados del uso empresarial de recursos materiales y financieros.

2. Para que la producción sea rentable, los productores fijan sus precios añadiendo un margen por encima del costo de producción. 
3. Los trabajadores no ahorran, y los perceptores del excedente gastan en consumo una cantidad fija $C^{A B}$ expresada en términos reales, más un porcentaje $b_{B}$ del excedente del periodo.

4. El gasto privado real en inversión ( $\left.I^{P}\right)$ lo tomaremos como dado, esto es, será una variable exógena.

El eje de este modelo es el principio de que, en tanto exista capacidad productiva disponible (trabajadores dispuestos a laborar al salario real vigente y bienes de capital instalados), en una economía mercantil capitalista el tamaño de producto total dependerá de la demanda agregada. La demanda agregada deberá ser tal que resulta posible vender la producción en condiciones de recuperar los costos y obtener el excedente que se deriva del margen aplicado sobre el costo al fijar el precio.

\section{NIVEL GENERAL DE PRECIOS}

Un esquema básico de fijación de precios de costo más margen por encima del costo puede ser representado, para el productor individual, de la siguiente manera:

$$
p=(c l+n)(1+\mu)
$$

donde $\mathrm{cl}$ es el costo laboral y $n$ el costo de los insumos materiales, ambos expresados por unidad de producto, mientras que $\mu$ es el margen por encima del costo unitario.

Ahora bien, sabemos que los insumos materiales de la ecuación anterior también fueron producidos con trabajo e insumos. Si descomponemos de esta manera todo el proceso de producción de un bien final hacia sus etapas previas, en las que se produjeron insumos, insumos de los insumos, etcétera, podemos establecer que su precio es igual al costo laboral y al excedente, acumulados a lo largo de todo el proceso. Es decir, el precio es igual al valor agregado total generado a lo largo de todas las etapas de su producción. Entonces, el precio se puede expresar como:

$$
p=\text { costo laboral acumulado }+ \text { excedente acumulado }
$$

Si tomamos al costo laboral acumulado y lo llamamos cla podemos anotar que la obtención del excedente implica que:

$$
\frac{p}{c l a}=(1+\theta)
$$


donde $\theta$ representa un margen implícito sobre el costo laboral acumulado, que surge a su vez de la obtención de excedentes en cada etapa de la producción del bien en cuestión.

Siguiendo la misma lógica, supongamos ahora que $W$ es el salario monetario promedio para toda la economía, $N$ el número de personas ocupadas (trabajando una jornada estándar), $Y$ el producto total real y $P$ el nivel general de precios. Naturalmente que la generación de un excedente para los productores implica que $P Y$, que es el valor monetario del producto total, será siempre mayor que el costo salarial total $W N$ y la diferencia estará en función de los márgenes que aplican los empresarios que siguen la práctica anteriormente descrita de fijación de sus precios. A la relación $P Y / W N$ la representaremos como $(1+m)$, definiendo a $m$ como el margen implícito por encima del costo laboral que surge a nivel macroeconómico. Pero para abreviar le llamaremos simplemente el margen de los productores.

Por lo anterior, el valor monetario del producto puede ser denotado como:

$$
P Y=W N \frac{P Y}{W N}=W N(1+m)
$$

Por lo que si despejamos al nivel de precios nos queda:

$$
P=\frac{W N}{Y}(1+m)=W \frac{N}{Y}(1+m)
$$

Ahora bien, el término $N / Y$ representa la cantidad de trabajo incorporado por unidad del producto total, lo cual no es otra cosa que el inverso de la productividad media del trabajo, o sea de $Y / N$. Si a este último concepto lo representamos como $z$, el nivel de precios puede quedar expresado de la siguiente forma:

$$
P=\frac{W}{z}(1+m)
$$

En la representación anterior, $W / z$ es el costo laboral promedio por unidad de producto final, reiterando que $W$ es el salario monetario promedio, $z$ la productividad media del trabajo y $m$ aparece ahora como el margen sobre el costo por unidad de producto. Recuérdese de lo dicho anteriormente que a nivel agregado los bienes intermedios desaparecen de la 
contabilidad macroeconómica, ya que el precio de cada bien o servicio de uso final se puede representar siempre como la suma del valor agregado total, en este caso la suma del costo laboral y del excedente que se generan a lo largo de todas las etapas de la producción.

El enfoque poskeynesiano adopta supuestos con referencia a la tecnología y a la estructura y funcionamiento de los mercados de trabajo y de bienes y servicios que, desde su posición, se considera que son más representativos de la realidad. En particular, y a diferencia del enfoque neoclásico, no considera la posibilidad de combinar en un momento dado trabajo y capital en proporciones variables, sino más bien en proporciones que tienden a ser fijas, establecidas por la tecnología. En este contexto, cada trabajador adicional será dotado con el equipo necesario para producir, para lo cual se asume que las empresas cuentan en tiempos normales con una capacidad instalada suficiente para ello. Por lo anterior, asumiremos la existencia de rendimientos constantes al trabajo, por lo que el valor de $z$ permanecerá igual a diferentes valores del producto total.

Por su parte, el salario monetario promedio no quedará establecido por un mercado competitivo e impersonal, sino como resultado de negociaciones obrero-patronales, y su valor dependerá de la capacidad negociadora de cada una de las partes, la cual está condicionada por diferentes factores institucionales y estructurales que configuran el mercado laboral. Finalmente, el tamaño del margen sobre el costo laboral será una expresión del poder de mercado de las empresas, el cual se corresponde con los niveles de concentración de la oferta entre un cierto número de productores prevalecientes en los diferentes mercados.

\section{NIVEL GENERAL DE PRECIOS Y DISTRIBUCIÓN DEL INGRESO}

La ecuación [6] del nivel de precios se puede reescribir de la siguiente manera:

$$
P=\frac{W}{z}+\frac{W}{z} m
$$

donde $P$, que es el valor monetario de una unidad de producto final, aparece separado en costo salarial y excedente.

Al dividir la ecuación anterior entre $P$, obtenemos la misma distribución, pero ahora expresada no en dinero, sino en términos de una unidad física de producto, esto es, en términos reales: 


$$
1=\frac{W}{P} \frac{1}{z}+\frac{W}{P} \frac{1}{z} m
$$

Podemos simplificar la ecuación anterior representando a $W / P$, que es el salario real, simplemente como $w$, lo que nos deja con:

$$
1=\frac{w}{z}+\frac{w}{z} m
$$

Esta última expresión nos indica que una unidad física de producto se separa entre el costo laboral real y el excedente real. Simplifiquemos aún más representando a $w / z$ como $d_{w}$ y a $(w / z) m$ como $d_{B}$, con lo que la ecuación se escribe ahora así:

$$
1=d_{W}+d_{B}
$$

Como la ecuación anterior está referida a una unidad física de producto, los términos $d_{W} y d_{B}$ fungen también como coeficientes (proporciones) de distribución entre remuneraciones laborales y excedente por unidad de producto.

Si ahora multiplicamos ambos lados por la productividad del trabajo, $z$, tenemos que lo que la economía produce por trabajador ocupado se reparte entre el salario real y el excedente de la siguiente manera:

$$
z=d_{w} z+d_{B} z
$$

Es decir, ahora estamos aplicando los coeficientes de distribución al producto por trabajador.

Ahora bien, ¿de dónde provienen los valores de los coeficientes de distribución? Estos están determinados por el margen por encima del costo. Veamos:

La participación de $d_{w}$ en una unidad física de producto es:

$$
\frac{d_{w}}{1}=d_{w}=\frac{\frac{w}{z}}{\frac{w}{z}+\frac{w}{z} m}=\frac{1}{1+m}
$$

mientras que la participación de $d_{B}$ es: 


$$
\frac{d_{B}}{1}=d_{B}=\frac{\frac{w}{z} m}{\frac{w}{z}+\frac{w}{z} m}=\frac{m}{1+m}
$$

En consecuencia, entre mayor sea el margen, menor será la participación de la remuneración laboral y más grande será la del excedente.

Si a continuación multiplicamos la ecuación [11] por el empleo total, $N$, tenemos que:

$$
z N=d_{w} z N+d_{B} z N
$$

pero como $z N$ es el producto real total de la economía, que es igual - por definición contable- al ingreso total, ambos representados por $Y$, tenemos que:

$$
Y=d_{W} Y+d_{B} Y
$$

donde $d_{W} Y$ representa las remuneraciones totales reales al trabajo y $d_{B} Y$ el excedente, también expresado en términos reales. Entonces, de la ecuación de los determinantes del nivel general de precios hemos derivado el perfil de la distribución del ingreso total. Remontándonos hacia atrás, hemos recorrido un camino que va de cómo las empresas fijan sus precios hasta la distribución del ingreso total entre remuneraciones laborales y excedente.

\section{DETERMINACIÓN DEL PRODUCTO (INGRESO) TOTAL}

Bajo los supuestos establecidos, la demanda agregada estará formada por: 1) el gasto en consumo $\left(C^{B}\right)$ y en inversión de los perceptores del excedente $\left(I^{P}\right)$, a quienes llamamos propietarios, y 2 ) el gasto en consumo de los asalariados $\left(C^{W}\right)$. De acuerdo con los supuestos, estos rubros de gasto se pueden expresar de la siguiente manera:

$$
\begin{gathered}
C^{B}=C^{A B}+b_{B} d_{B} Y \\
I^{P}=I^{P} \\
C^{W}=d_{W} Y
\end{gathered}
$$

de modo que, igualando al flujo de producto-ingreso (repartido entre salarios y excedente) con la demanda agregada tenemos que: 


$$
d_{W} Y+d_{B} Y=C^{A B}+b_{B} d_{B} Y+I^{P}+d_{W} Y
$$

donde al resolver para $Y$ nos queda:

$$
Y=\frac{1}{\left(1-b_{B}\right) d_{B}}\left(C^{A B}+I^{P}\right)
$$

Si ahora, a partir del análisis de la distribución del ingreso, recordamos que $d_{B}=1-d_{W}$, la ecuación [19] se puede reescribir así:

$$
Y=\frac{1}{\left(1-b_{B}\right)\left(1-d_{W}\right)}\left(C^{A B}+I^{P}\right)
$$

De la ecuación [20] podemos establecer que el producto total depende del gasto en consumo y en inversión de los propietarios, así como de la distribución del ingreso, expresada en dicha ecuación como la participación de los salarios en el ingreso.

De manera más puntual, podemos establecer de la ecuación [20] que el producto será más elevado entre:

1. más gasten en consumo (variables $C^{A B}$ y $b_{B}$ ) y en inversión $\left(I^{P}\right)$ los propietarios, y

2. mayor sea la participación de los salarios en el ingreso $\left(d_{w}\right)$, ya que hemos supuesto que los asalariados gastan todo su ingreso en consumo, mientras que los propietarios ahorran una parte del excedente.

\section{DETERMINANTES DEL EXCEDENTE}

De la ecuación [18] podemos también obtener una solución para el excedente, $d_{B} Y$. Despejando ese término nos queda:

$$
d_{B} Y=\frac{1}{1-b_{B}}\left(C^{A B}+I^{P}\right)
$$

Esto es, que el excedente depende de lo que gasten los propietarios en su conjunto en consumo y en inversión. Entre mayores sean estos gastos más grande será el ingreso total de los propietarios.

De hecho, lo anterior se puede ver de una manera más simple y directa. Tomemos de nuevo la ecuación [18].

Pasando a $d_{w}$ del lado izquierdo, nos queda que: 


$$
d_{B} Y=C^{A B}+b_{B} d_{B} Y+l^{P}
$$

Es decir, que el excedente es igual, contablemente, al gasto de los propietarios. Pero la ecuación [18], recuérdese, la interpretamos en el sentido de que la demanda agregada (lado derecho) determina al producto (lado izquierdo). Entonces, manteniendo la misma relación de causalidad, tenemos que es el gasto de los propietarios el que determina al excedente.

La representación gráfica de la determinación del producto se muestra en la gráfica 1. La línea punteada de 45 grados representa puntos en donde la demanda agregada $(D)$ es igual al producto. La función de demanda agregada corta la línea de 45 grados al nivel de producto $Y_{1}$. La gráfica muestra también una función para el excedente, $d_{B} Y$, a cualquier nivel de producto. Al nivel de producto $Y_{1}$, al proyectar el punto sobre la recta $d_{B} Y$ a la línea de 45 grados, podemos marcar la magnitud correspondiente sobre el mismo eje del producto. La diferencia $Y-d_{B} Y_{1}$ corresponde a los salarios totales.

\section{GRÁFICA 1}

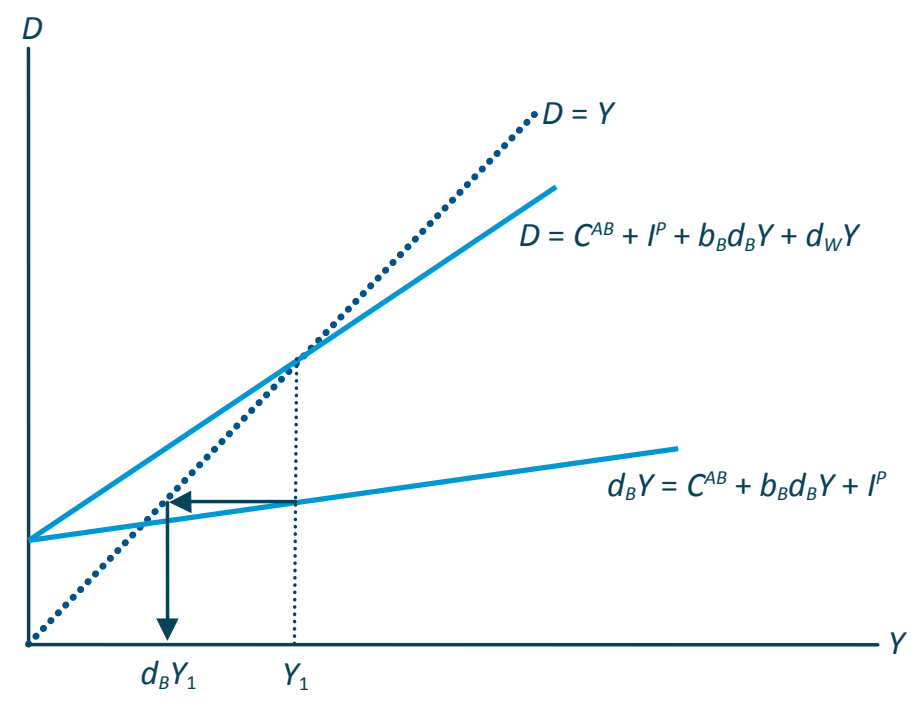

\section{EMPLEO Y DESEMPLEO}

Una vez conocido el producto total, y dada la productividad del trabajo, $z$, podemos establecer el empleo total, $N$, y la tasa de desempleo $(u)$. El empleo será igual a: 


$$
N=\frac{Y}{Z}
$$

mientras que si $L$ es la población económicamente activa, la tasa de desempleo será:

$$
u=\frac{L-N}{L}=\frac{L-\frac{Y}{Z}}{L}=1-\frac{1}{L Z} Y
$$

Entonces, la tasa de desempleo es una función inversa del producto total y función directa tanto de la productividad del trabajo como del tamaño de la población económicamente activa.

\section{UN AUMENTO EN EL GASTO DE LOS PROPIETARIOS}

La gráfica 2 nos muestra el efecto de un cambio en $I^{P}$ y en $C^{A B}$, los rubros autónomos de gasto de los propietarios. Obsérvese que el intercepto de la función de demanda se traslada hacia arriba, y que la nueva intersección con la línea de 45 grados nos muestra que el nuevo nivel del producto, ahora $Y_{2}$, es mayor que el aumento en el gasto autónomo de los propietarios. Esto, naturalmente, es la expresión del efecto multiplicador. Al aumentar $Y$ también lo hacen el gasto en consumo inducido de los propietarios $\left(b_{B} d_{B} \Delta Y\right)$ y el de los asalariados que se incorporan al empleo $\left(d_{w} \Delta Y\right)$. El excedente crece en la medida en que aumenta el gasto total de los propietarios.

\section{UN AUMENTO EN EL MARGEN SOBRE EL COSTO LABORAL}

La gráfica 3 nos muestra este caso. Al aumentar el margen sobre el costo laboral se reduce la participación de los salarios en el ingreso total y se incrementa en esa misma medida la participación del excedente. De acuerdo con los supuestos del modelo, en cuanto a las propensiones al ahorro de las dos clases de perceptores, el gasto total en consumo se reduce y con ello la demanda agregada se abate. Obsérvese que el nuevo nivel de producto es ahora $Y_{0}$. Con ello evidentemente que habría caído el empleo.

En este caso el excedente se mantiene igual. Los propietarios reciben una mayor participación de un ingreso total proporcionalmente más pequeño. Una cosa compensa exactamente a la otra. 


\section{GRÁFICA 2}

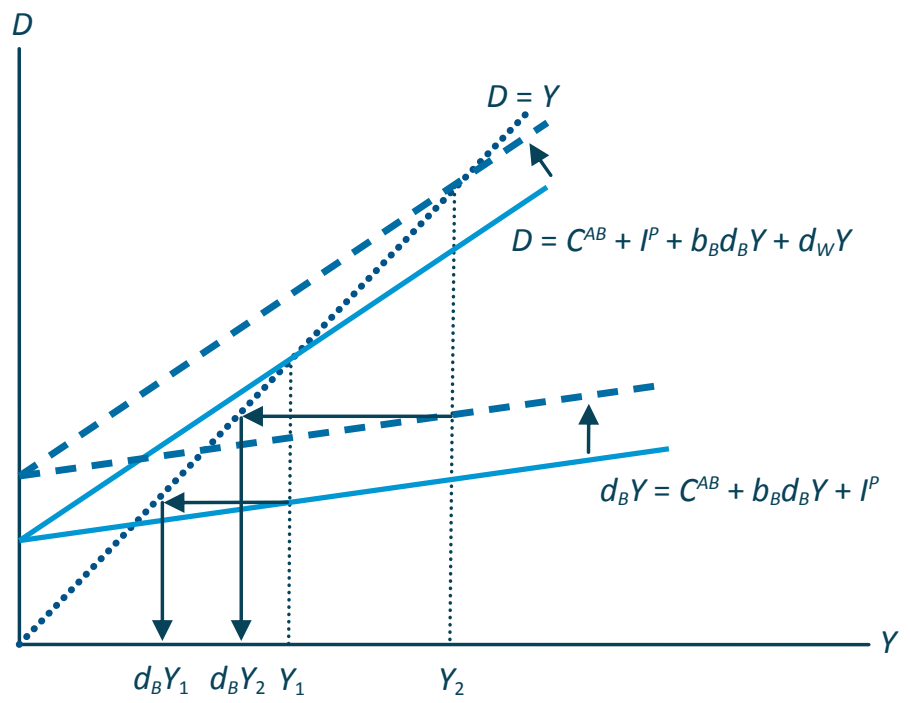

\section{GRÁFICA 3}

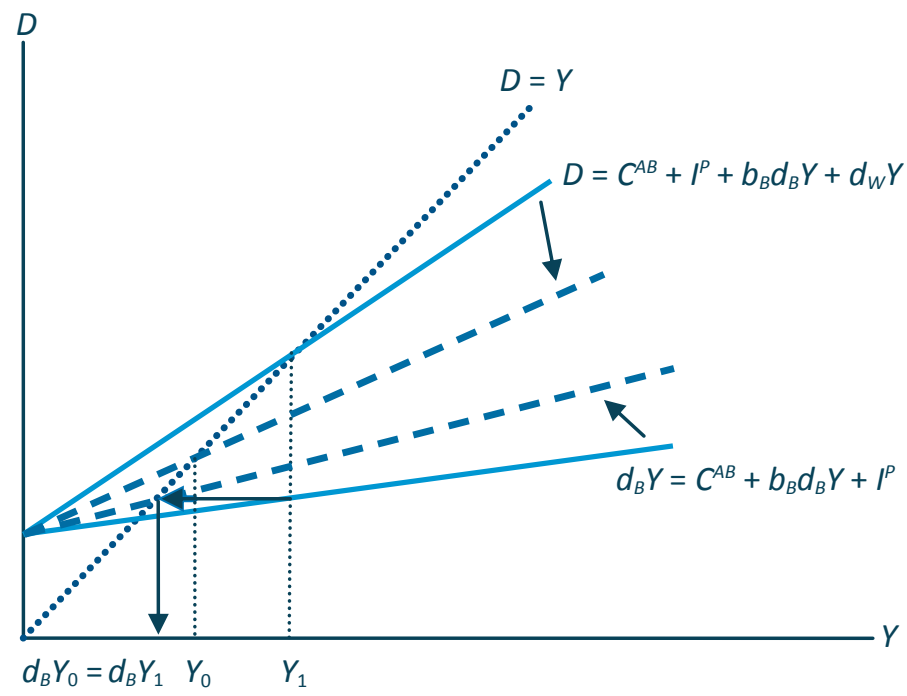

\section{SALARIO REAL Y EMPLEO}

Partimos de la ecuación que nos indica que el producto total es igual a la demanda agregada: 


$$
Y=C^{W}+C^{B}+I^{P}
$$

Pero si:

$Y=z N$

$C^{W}=w N$ (los perceptores de ingresos laborales no ahorran)

$C^{B}=C^{A B}+b_{B}(z N-w N)$, donde $z N-w N$ es el excedente

La ecuación puede ser reescrita de la siguiente manera:

$$
z N=w N+C^{A B}+b_{B}(z N-w N)+l^{P}
$$

Ecuación que si resolvemos para el nivel de empleo nos da:

$$
N=\frac{C^{A B}+l^{P}}{(z-w)\left(1-b_{B}\right)}
$$

La expresión anterior nos indica que, dado todo lo demás constante, el empleo es una función directa del salario real. Su representación gráfica aparece en la gráfica 4. De la ecuación [25] y su correspondiente representación gráfica se desprende que el empleo será mayor si aumentan $C A^{B}, b_{B}$ e $I^{P}$, es decir, el gasto de los propietarios en consumo e inversión. Por otra parte, el empleo será menor a cada nivel de salario real si la productividad del trabajo es más elevada.

Esta relación entre salario real y empleo es la opuesta del modelo neoclásico que se presenta habitualmente en los manuales de economía, en donde para que los productores contraten más trabajadores el salario real tiene que bajar. Las razones de la diferencia son:

1. En el modelo neoclásico la demanda agregada de bienes de consumo no se relaciona con la distribución del ingreso, en el poskeynesiano sí, al suponer que los perceptores del ingreso tienen diferentes propensiones marginales a consumir. El salario real, en particular, si bien es un costo, también es una fuente de demanda efectiva.

2. En el modelo neoclásico hay rendimientos decrecientes al empleo del trabajo y el punto de maximización de ganancias aparece cuando el salario real es igual a productividad marginal (decreciente) del trabajo. En el modelo poskeynesiano no hay punto de maximización de ganancias. Cada productor agrega un margen por encima del costo $y$, dado ese margen, gana más entre más venda. 


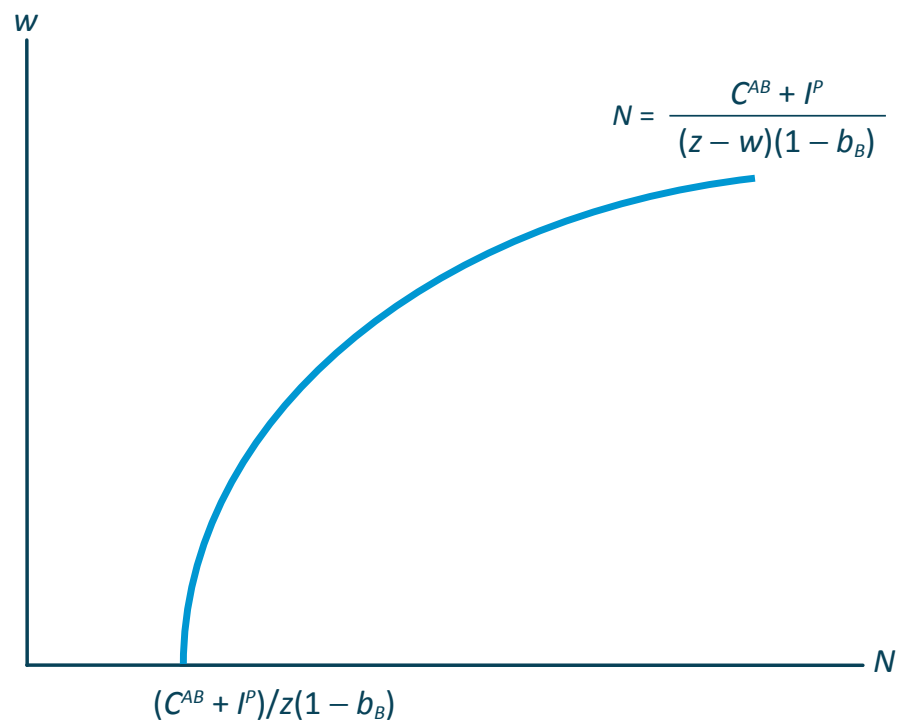

\section{ANOTACIONES FINALES}

El modelo básico aquí desarrollado nos proporciona un escenario macroeconómico sumamente simplificado, pero a pesar de ello nos muestra relaciones de causalidad muy importantes para comprender la determinación del producto, el empleo y el tamaño del excedente. Estas relaciones se amplían si extendemos el modelo añadiendo la tributación y el gasto público, así como las transacciones con el exterior contenidas en la cuenta corriente de la balanza de pagos.

En primer lugar podemos mencionar, sin mostrarlo en el presente trabajo, que la demanda agregada y el excedente se verían impulsados tanto por el exceso de gasto público por encima de la tributación como por el exceso de exportaciones por encima de las importaciones. En cualquier caso, la distribución del ingreso continuaría siendo un elemento central en la determinación del tamaño de la demanda agregada en tanto la propensión al consumo correspondiente al gasto de los ingresos salariales sea mayor que la que corresponde al excedente. La distribución primaria del ingreso se vería modificada por las variables fiscales y al incorporar los pagos factoriales y las transferencias del exterior, todo esto al pasar del ingreso total al ingreso nacional privado disponible. Por otra parte, si el ahorro de los asalariados es positivo, ello le resta al tamaño del excedente. 
Por lo que toca a la relación entre el salario real y el empleo, su asociación positiva en principio se sostiene, pero se puede llegar a invertir cuando un aumento en el salario real se relaciona con la reducción de la competitividad de los productos de la economía en el comercio internacional, y si a partir de esto último la reacción de las exportaciones y las importaciones es suficientemente intensa como para generar un efecto contraccionista sobre la demanda agregada.

\section{REFERENCIAS}

Ibarra, J., 2000. Producto, empleo, distribución y ganancias. Un enfoque poskeynesiano de corto plazo. México: Facultad de Economía, UNAM. Kalecki, M., 1977. Ensayos escogidos sobre la dinámica de la economía capitalista. México: Fondo de Cultura Económica (FCE).

Lavoie, M., 2006. Introduction to Post Keynesian Economics. Estados Unidos: Palgrave Macmillan.

López, J. y Assouz, M., 2010. Michael Kalecki. Estados Unidos: Palgrave Macmillan. 